\title{
SECOND-DERIVATIVE ESTIMATES FOR SOLUTIONS OF TWO-DIMENSIONAL MONGE-AMPĖRE EQUATIONS
}

\author{
FRIEDMAR SCHULZ
}

(Communicated by Barbara L. Keyfitz)

\begin{abstract}
Heinz-Lewy type a priori estimates are derived for the absolute values of the second derivatives of solutions $z(x, y) \in C^{1,1}(\Omega)$ of MongeAmpère equations of the general form

$$
A r+2 B s+C t+\left(r t-s^{2}\right)=E
$$

in the interior of the domain $\Omega$. The coefficients $A, B, C, E$ depend in particular on the gradient of $z(x, y)$ and satisfy certain structural conditions.
\end{abstract}

\section{INTRODUCTION AND STATEMENT OF THE RESULT}

Consider a solution $z=z(x, y) \in C^{1,1}(\Omega)$ of the elliptic Monge-Ampère equation

$$
A r+2 B s+C t+\left(r t-s^{2}\right)=E
$$

or, equivalently,

$$
(r+C)(t+A)-(s-B)^{2}=\Delta,
$$

where

$$
\Delta=A C-B^{2}+E>0,
$$

and assume without loss of generality that

$$
t+A>0 \text {. }
$$

The coefficients $A, B, C$ and the nonhomogeneous term $E$ depend on the five variables $x, y, z, p, q$. Here $p, q, r, s, t$ represent the first and second derivatives of $z(x, y)$,

$$
p=z_{x}, q=z_{y} ; r=z_{x x}, s=z_{x y}, t=z_{y y} .
$$

Received by the editors August 3, 1989.

1980 Mathematics Subject Classification (1985 Revision). Primary 35A30, 35B45, 34D10, 35J15, 35J45, 35J60; Secondary 30F10.

Key words and phrases. Fully nonlinear elliptic equations, Monge-Ampère equations, quasilinear elliptic systems, Heinz-Lewy systems, regularity, a priori estimates, characteristics, uniformization, conformal mappings, convex surfaces.

Most of the research for this article was carried out during the Fall of 1988, when I visited the University of Kentucky in Lexington. I gratefully acknowledge the support by NSF grant RII8610671 and the Commonwealth of Kentucky through the Kentucky EPSCoR Program. 
Assumption (A). The solution $z(x, y)$ satisfies the estimate

$$
|D z| \leq K_{1} \text {. }
$$

Assumption (B). Suppose that $A, B, C \in C^{1}\left(\Omega \times \mathbb{R}^{3}\right)$, and let

$$
\Delta=K(x, y, z) D(x, y, z, p, q),
$$

with $D \in C^{1}\left(\Omega \times \mathbb{R}^{3}\right)$ and $K \in C^{\mu}(\Omega \times \mathbb{R})$ for some $\mu, 0<\mu<1$, such that

$$
\begin{aligned}
|A|,|B|,|C|,|D|,|K| & \leq a, \\
\left|A_{x}\right|, \ldots,\left|D_{q}\right| & \leq b, \\
{[K]_{\mu}^{\Omega \times \mathbb{R}} } & \leq b^{\prime}, \\
D, K & \geq \frac{1}{c} .
\end{aligned}
$$

Assumption (C). Suppose that the functions

$$
\begin{aligned}
& \phi_{1}(x, y)=A_{p}, \\
& \phi_{2}(x, y)=A_{q}+2 B_{p}, \\
& \phi_{3}(x, y)=2 B_{q}+C_{p}, \\
& \phi_{4}(x, y)=C_{q}
\end{aligned}
$$

belong to the Lipschitz class $C^{0,1}(\Omega)$ and satisfy the conditions

$$
\left[\phi_{1}\right]_{0,1}^{\Omega}, \ldots,\left[\phi_{4}\right]_{0,1}^{\Omega} \leq d
$$

The main result of this note then reads as follows:

Theorem 1. The solution $z(x, y)$ belongs to the Hölder class $C_{\mathrm{loc}}^{2, \mu}(\Omega)$. For each subset $\Omega^{\prime}$ which is compactly contained in $\Omega$, there is a second-derivative estimate of the form

$$
\left\|D^{2} z\right\|_{C^{\mu}\left(\Omega^{\prime}\right)} \leq C,
$$

where the constant $C$ depends only on $\mu, a, b, b^{\prime}, c, d, K_{1}$, and $\operatorname{dist}\left(\Omega^{\prime}, \partial \Omega\right)$.

The regularity part of the theorem and the Hölder estimate

$$
\left[D^{2} z\right]_{\mu}^{\Omega^{\prime}} \leq C
$$

have been derived in [7,9] if the coefficients $A, B, C, E$ are $\mu$-Hölder continuous with respect to the variables $x, y, z, p, q$. This was shown by invoking the Legendre-like transformation

$$
\begin{aligned}
& u=x, \\
& v=q(x, y) .
\end{aligned}
$$


The purpose of the present article is to provide sharp local estimates also for the absolute values of the second derivatives of $z(x, y)$. The present approach consists of introducing characteristic parameters; i.e., of constructing a conformal map $(u(x, y), v(x, y))$ with respect to the characteristic form

$$
(r+C) d x^{2}+2(s-B) d x d y+(t+A) d y^{2} .
$$

This approach is due to H. Lewy [6] and E. Heinz [1, 3], who also constructed a counterexample showing that Monge-Ampère equations of the form (1) in general do not admit second-derivative estimates for their solutions. Heinz [3] discovered the structural conditions of Assumption (C) and derived secondderivative estimates under additional regularity assumptions regarding both the solution $z(x, y)$ and the coefficients $A, B, C, E$.

The present estimates rest on sharp estimates for the Jacobian of a certain quasilinear elliptic second-order system which is satisfied by the inverse mapping $(x(u, v), y(u, v))$. These estimates were derived in [8], generalizing classical theorems of Lewy [5] and Heinz [2, 4]. An improved version is stated as Proposition 4.

The results of the present article are tailor-made for application to prescribed curvature equations, in particular to the prescribed Gauss curvature equation,

$$
r t-s^{2}=K(x, y, z)\left(1+p^{2}+q^{2}\right)^{2},
$$

and to the Darboux equation, which in terms of local parameters $u, v$ for a convex surface takes the form

$$
\begin{aligned}
\left(\rho_{u u}\right. & \left.-\Gamma_{11}^{1} \rho_{u}-\Gamma_{11}^{2} \rho_{v}\right)\left(\rho_{v v}-\Gamma_{22}^{1} \rho_{u}-\Gamma_{22}^{2} \rho_{v}\right)-\left(\rho_{u v}-\Gamma_{12}^{1} \rho_{u}-\Gamma_{12}^{2} \rho_{v}\right)^{2} \\
& =K(u, v)\left(E G-F^{2}-G \rho_{u}^{2}+2 F \rho_{u} \rho_{v}-E \rho_{v}^{2}\right) .
\end{aligned}
$$

Here $\rho=\rho(u, v)$ represents any component of the radius vector of the surface. The expressions $\Gamma_{i j}^{k}$ are the Christoffel symbols of the first fundamental form

$$
E(u, v) d u^{2}+2 F(u, v) d u d v+G(u, v) d v^{2},
$$

and $K(u, v)$ denotes the Gauss curvature.

\section{CHARACTERISTIC PARAMETERS}

Lemma 2. Let $A, B, C, D$ be functions of class $C^{1}(\Omega)$, and let $z \in C^{2, \mu}(\Omega)$ for some $\mu, 0<\mu<1$, be a solution of the Monge-Ampere equation (2) such that

$$
\Delta=K(x, y) D(x, y), \quad K, D>0 .
$$

Let $\bar{B}_{R}=\bar{B}_{R}\left(x_{0}, y_{0}\right)=\left\{\left(x-x_{0}\right)^{2}+\left(y-y_{0}\right)^{2} \leq R^{2}\right\} \subset \Omega$. Then there exists a homeomorphism $(x, y)=(x(u, v), y(u, v))$ from $\bar{B}=\left\{u^{2}+v^{2} \leq 1\right\}$ onto $\bar{B}_{R}$ 
of class $C_{\mathrm{loc}}^{1, \mu}(B)$ with $x(0,0)=x_{0}, y(0,0)=y_{0}$, which satisfies the system

$$
\begin{aligned}
& L_{K} x=\frac{1}{\sqrt{D}}\left(A_{x}+B_{y}-(t+A) \frac{D_{x}}{2 D}+(s-B) \frac{D_{y}}{2 D}\right) D x \wedge D y, \\
& L_{K} y=\frac{1}{\sqrt{D}}\left(B_{x}+C_{y}+(s-B) \frac{D_{x}}{2 D}-(r+C) \frac{D_{y}}{2 D}\right) D x \wedge D y .
\end{aligned}
$$

Here

$$
L_{K}=\frac{\partial}{\partial u}\left(\sqrt{K} \frac{\partial}{\partial u}\right)+\frac{\partial}{\partial v}\left(\sqrt{K} \frac{\partial}{\partial v}\right)
$$

Furthermore,

$$
D x \wedge D y=x_{u} y_{v}-x_{v} y_{u} \neq 0
$$

and

$$
\begin{aligned}
\frac{t+A}{\sqrt{\Delta}} & =\frac{|D x|^{2}}{D x \wedge D y}, \\
-\frac{s-B}{\sqrt{\Delta}} & =\frac{D x \cdot D y}{D x \wedge D y} \\
\frac{r+C}{\sqrt{\Delta}} & =\frac{|D y|^{2}}{D x \wedge D y}
\end{aligned}
$$

Proof. Consider the characteristic form

$$
\begin{aligned}
d s^{2} & =(r+C) d x^{2}+2(s-B) d x d y+(t+A) d y^{2} \\
& =a d x^{2}+2 b d x d y+c d y^{2}
\end{aligned}
$$

in the disc $\bar{B}_{R}$. Uniformizing parameters $(u, v)$ in the disc $\bar{B}=\left\{u^{2}+v^{2} \leq 1\right\}$ can be introduced since the coefficients $a, b, c$ of the metric $d s^{2}$ belong to the Hölder class $C^{\mu}(\Omega)$ : There exists a homeomorphism $(u, v)=(u(x, y)$, $v(x, y))$ from $\bar{B}_{R}$ onto $\bar{B}$ of class $C_{\text {loc }}^{1, \mu}\left(B_{R}\right)$ with $u\left(x_{0}, y_{0}\right)=0, v\left(x_{0}, y_{0}\right)=$ 0 , which solves the Beltrami system

$$
\begin{aligned}
& \sqrt{\Delta} u_{x}=-b v_{x}+a v_{y}, \\
& \sqrt{\Delta} u_{y}=-c v_{x}+b v_{y} .
\end{aligned}
$$

The system for the inverse mapping $(x, y)=(x(u, v), y(u, v))$ reads as

$$
\begin{gathered}
\sqrt{\Delta} x_{u}=b x_{v}+c y_{v}, \\
\sqrt{\Delta} x_{v}=-b x_{u}-c y_{u},
\end{gathered}
$$

resp.,

$$
\begin{gathered}
\sqrt{\Delta} y_{u}=-a x_{v}-b y_{v}, \\
\sqrt{\Delta} y_{v}=a x_{u}+b y_{u} .
\end{gathered}
$$


The equivalent conformality relations are

$$
\begin{gathered}
|D x|^{2}=\lambda c, \\
D x \cdot D y=-\lambda b, \\
|D y|^{2}=\lambda a,
\end{gathered}
$$

where

$$
\lambda=\frac{D x \wedge D y}{\sqrt{\Delta}} .
$$

The relations $(12,13,14)$ are identical to the statements $(5,6,7)$.

In order to obtain the system $(3,4)$, one differentiates the systems $(8,9)$ and $(10,11)$ :

$$
\begin{aligned}
L_{K} x & =\left(\frac{b}{\sqrt{D}}\right)_{u} x_{v}+\left(\frac{c}{\sqrt{D}}\right)_{u} y_{v}-\left(\frac{b}{\sqrt{D}}\right)_{v} x_{u}-\left(\frac{c}{\sqrt{D}}\right)_{v} y_{u} \\
& =\left(\left(\frac{c}{\sqrt{D}}\right)_{x}-\left(\frac{b}{\sqrt{D}}\right)_{y}\right)_{u}\left(x_{u} y_{v}-x_{v} y_{u}\right) \\
& =\frac{1}{\sqrt{D}}\left(A_{x}+B_{y}-(t+A) \frac{D_{x}}{2 D}+(s-B) \frac{D_{y}}{2 D}\right) D x \wedge D y, \\
L_{K} y & =-\left(\frac{a}{\sqrt{D}}\right)_{u} x_{v}-\left(\frac{b}{\sqrt{D}}\right)_{u} y_{v}+\left(\frac{a}{\sqrt{D}}\right)_{v} x_{u}+\left(\frac{b}{\sqrt{D}}\right)_{v} y_{u} \\
& =\left(\left(\frac{a}{\sqrt{D}}\right)_{y}-\left(\frac{b}{\sqrt{D}}\right)_{x}\right)_{\left(x_{u} y_{v}-x_{v} y_{u}\right)} \\
& =-\frac{1}{\sqrt{D}}\left(B_{x}+C_{y}+(s-B) \frac{D_{x}}{2 D}-(r+C) \frac{D_{y}}{2 D}\right) D x \wedge D y .
\end{aligned}
$$

Here one first assumes that $z(x, y) \in C^{3}(\Omega)$ and then removes this restriction by approximation. This is possible because the Dirichlet integrals of the approximating maps are bounded in view of the conformality relations $(5,7)$. The system $(3,4)$ and the system

$$
\begin{aligned}
& \frac{\partial}{\partial x}\left(\frac{c u_{x}-b u_{y}}{\sqrt{\Delta}}\right)+\frac{\partial}{\partial y}\left(\frac{-b u_{x}+a u_{y}}{\sqrt{\Delta}}\right)=0, \\
& \frac{\partial}{\partial x}\left(\frac{c v_{x}-b v_{y}}{\sqrt{\Delta}}\right)+\frac{\partial}{\partial y}\left(\frac{-b v_{x}+a v_{y}}{\sqrt{\Delta}}\right)=0
\end{aligned}
$$

therefore each allow interior $C^{1, \mu}$-estimates for their solutions. 
Lemma 3. Suppose that $A, B, C, D \in C^{1}\left(\Omega \times \mathbb{R}^{3}\right)$. The mapping $(x, y)=$ $(x(u, v), y(u, v))$ from Lemma 2 then solves the system

$$
\begin{aligned}
& \frac{1}{\sqrt{K}} L_{K} x=h_{1}|D x|^{2}+h_{2} D x \cdot D y+h_{3}|D y|^{2}+h_{4} D x \wedge D y, \\
& \frac{1}{\sqrt{K}} L_{K} y=\tilde{h}_{1}|D x|^{2}+\tilde{h}_{2} D x \cdot D y+\tilde{h}_{3}|D y|^{2}+\tilde{h}_{4} D x \wedge D y,
\end{aligned}
$$

where

$$
\begin{aligned}
& h_{1}(x, y)=B_{q}-\frac{1}{2 D}\left(D_{x}+D_{z} p-D_{p} C+D_{q} B\right), \\
& h_{2}(x, y)=-A_{q}-B_{p}-\frac{1}{2 D}\left(D_{y}+D_{z} q+D_{p} B-D_{q} A\right), \\
& h_{3}(x, y)=A_{p}, \\
& h_{4}(x, y)=\frac{1}{\sqrt{\Delta}}\left(A_{x}+A_{z} p+B_{y}+B_{z} q-A_{p} C+\left(A_{q}+B_{p}\right) B-B_{q} A-\frac{D_{p} K}{2}\right), \\
& \tilde{h}_{1}(x, y)=C_{q}, \\
& \tilde{h}_{2}(x, y)=-B_{q}-C_{p}-\frac{1}{2 D}\left(D_{x}+D_{z} p-D_{p} C+D_{q} B\right), \\
& \tilde{h}_{3}(x, y)=B_{p}-\frac{1}{2 D}\left(D_{y}+D_{z} q+D_{p} B-D_{q} A\right), \\
& \tilde{h}_{4}(x, y)=\frac{1}{\sqrt{\Delta}}\left(B_{x}+B_{z} p+C_{y}+C_{z} q-B_{p} C+\left(B_{q}+C_{p}\right) B-C_{q} A-\frac{D_{q} K}{2}\right) .
\end{aligned}
$$

Proof. Compute

$$
\begin{aligned}
\frac{\partial A}{\partial x}+ & \frac{\partial B}{\partial y}-\frac{t+A}{2 D} \frac{\partial D}{\partial x}+\frac{s-B}{2 D} \frac{\partial D}{\partial y} \\
= & A_{x}+A_{z} p+B_{y}+B_{z} q-A_{p} C+\left(A_{q}+B_{p}\right) B-B_{q} A \\
& +A_{p}(r+C)+\left(A_{q}+B_{p}\right)(s-B)+B_{q}(t+A) \\
& -\frac{t+A}{2 D}\left(D_{x}+D_{z} p-D_{p} C+D_{q} B+D_{p}(r+C)+D_{q}(s-B)\right) \\
& +\frac{s-B}{2 D}\left(D_{y}+D_{z} q+D_{p} B-D_{q} A+D_{p}(s-B)+D_{q}(t+A)\right) \\
= & A_{x}+A_{z} p+B_{y}+B_{z} q-A_{p} C+\left(A_{q}+B_{p}\right) B-B_{q} A \\
& +(t+A)\left(B_{q}-\frac{1}{2 D}\left(D_{x}+D_{z} p-D_{p} C+D_{q} B\right)\right) \\
& +(s-B)\left(A_{q}+B_{p}+\frac{1}{2 D}\left(D_{y}+D_{z} q+D_{p} B-D_{q} A\right)\right) \\
& +(r+C) A_{p}-\frac{D_{p}}{2 D}\left((t+A)(r+C)-(s-B)^{2}\right) \\
= & -\frac{\sqrt{\Delta}}{J(x, y)}\left(h_{1}|D x|^{2}+h_{2} D x \cdot D y+h_{3}|D y|^{2}+h_{4} D x \wedge D y\right) .
\end{aligned}
$$




$$
\begin{aligned}
\frac{\partial B}{\partial x}+ & \frac{\partial C}{\partial y}+\frac{s-B}{2 D} \frac{\partial D}{\partial x}-\frac{r+C}{2 D} \frac{\partial D}{\partial y} \\
= & B_{x}+B_{z} p+C_{y}+C_{z} q-B_{p} C+\left(B_{q}+C_{p}\right) B-C_{q} A \\
& +B_{p}(r+C)+\left(B_{q}+C_{p}\right)(s-B)+C_{q}(t+A) \\
& +\frac{s-B}{2 D}\left(D_{x}+D_{z} p-D_{p} C+D_{q} B+D_{p}(r+C)+D_{q}(s-B)\right) \\
& -\frac{r+C}{2 D}\left(D_{y}+D_{z} q+D_{p} B-D_{q} A+D_{p}(s-B)+D_{q}(t+A)\right) \\
= & B_{x}+B_{z} p+C_{y}+C_{z} q-B_{p} C+\left(B_{q}+C_{p}\right) B-C_{q} A \\
& +(t+A) C_{q} \\
& +(s-B)\left(B_{q}+C_{p}+\frac{1}{2 D}\left(D_{x}+D_{z} p-D_{p} C+D_{q} B\right)\right) \\
& +(r+C)\left(B_{p}-\frac{1}{2 D}\left(D_{y}+D_{z} q+D_{p} B-D_{q} A\right)\right) \\
& -\frac{D_{q}}{2 D}\left((r+C)(t+A)-(s-B)^{2}\right) \\
= & -\frac{\sqrt{\Delta}}{J(x, y)}\left(\tilde{h}_{1}|D x|^{2}+\tilde{h}_{2} D x \cdot D y+\tilde{h}_{3}|D y|^{2}+\tilde{h}_{4} D x \wedge D y\right)
\end{aligned}
$$

\section{Univalent Solutions of Heinz-Lewy TyPE SyStems}

Consider a solution $(x, y)=(x(u, v), y(u, v))$ of class $W^{1,2}(B), B=$ $\left\{u^{2}+v^{2}<1\right\}$, of the Heinz-Lewy type system:

$$
L x=h_{1}(x, y)|D x|^{2}+h_{2}(x, y) D x \cdot D y+h_{3}(x, y)|D y|^{2}+h_{4}(x, y) D x \wedge D y,
$$

$$
L y=\tilde{h}_{1}(x, y)|D x|^{2}+\tilde{h}_{2}(x, y) D x \cdot D y+\tilde{h}_{3}(x, y)|D y|^{2}+\tilde{h}_{4}(x, y) D x \wedge D y,
$$

where

$$
L=\frac{1}{a(u, v, x, y)}\left(\frac{\partial}{\partial u}\left(a(u, v, x, y) \frac{\partial}{\partial u}\right)+\frac{\partial}{\partial v}\left(a(u, v, x, y) \frac{\partial}{\partial v}\right)\right) .
$$

The following assumptions are imposed on the coefficients $a, h_{1}, \ldots, \tilde{h}_{4}$ : Assumption (D). The function $a=a(u, v, x, y)$ belongs to the Hölder class $C^{\mu}\left(B \times \mathbb{R}^{2}\right)$ for some $\mu, 0<\mu<1$, such that

$$
0<\lambda \leq a(u, v, x, y) \leq \Lambda<+\infty
$$

for all $(u, v) \in B,(x, y) \in \mathbb{R}^{2}$, and

$$
[a]_{\mu}^{B \times \mathbb{R}^{2}} \leq L
$$


Assumption (E). The coefficients $h_{1}, \ldots, \tilde{h}_{4}$ are bounded, Borel-measurable functions on $\mathbb{R}^{2}$ such that

$$
\left|h_{1}(x, y)\right|, \ldots,\left|\tilde{h}_{4}(x, y)\right| \leq M_{0}
$$

for all $(x, y) \in \mathbb{R}^{2}$, and the functions

$$
\begin{aligned}
& \omega_{1}(x, y)=\tilde{h}_{1}(x, y), \\
& \omega_{2}(x, y)=h_{1}(x, y)-\tilde{h}_{2}(x, y), \\
& \omega_{3}(x, y)=h_{2}(x, y)-\tilde{h}_{3}(x, y), \\
& \omega_{4}(x, y)=h_{3}(x, y)
\end{aligned}
$$

$\left((x, y) \in \mathbb{R}^{2}\right)$ are Lipschitz continuous with Lipschitz constant $\leq M_{1}$ :

$$
\left[\omega_{1}\right]_{0,1}^{\mathbb{R}^{2}}, \ldots,\left[\omega_{4}\right]_{0,1}^{\mathbb{R}^{2}} \leq M_{1}
$$

Assumption (F). Suppose that the Dirichlet integral of the solution mapping $(x, y)$ satisfies the estimate

$$
\int_{B}\left(|D x|^{2}+|D y|^{2}\right) d u d v \leq N
$$

Proposition 4. Let $(x, y)=(x(u, v), y(u, v))$ be a homeomorphism from the closed unit disc $\bar{B}$ onto $\bar{B}_{R}\left(x_{0}, y_{0}\right)=\left\{\left(x-x_{0}\right)^{2}+\left(y-y_{0}\right)^{2} \leq R^{2}\right\}$ of class $W^{1,2}$ which solves the system $(15,16)$. Suppose that the Assumptions (D, E, F) are satisfied, and assume that $x(0,0)=x_{0}, y(0,0)=y_{0}$.

Then $(x, y) \in C_{\mathrm{loc}}^{1, \mu}(B)$, and the Jacobian $D x \wedge D y$ does not vanish in $B$. The following estimates hold for all $B_{\rho}=\left\{u^{2}+v^{2}<\rho^{2}\right\}, 0<\rho<1$ :

$$
\begin{gathered}
\|x, y\|_{C^{1, \mu}\left(B_{\rho}\right)} \leq C, \\
D x \wedge D y \geq c>0,
\end{gathered}
$$

where the constants $C, c$ depend only on $\mu, \lambda, \Lambda, L, M_{0}, M_{1}, N, \rho$, and $R$.

Proposition 4 was proved in [8] under the assumption that all coefficients $h_{1}, \ldots, \tilde{h}_{4}$ are Lipschitz continuous. It was noted, however, at the end of [8] that the proof, which is based on [2], requires only the Lipschitz continuity of the combinations $\omega_{1}, \ldots, \omega_{4}$.

\section{Proof of THEOREM 1}

The regularity $z(x, y) \in C^{2, \mu}(\Omega)$ follows from [9]. The mapping $(x, y)$ from Lemma 2 therefore satisfies, by Lemma 3, a system of the form $(15,16)$. 
In order to apply Proposition 4, estimate

$$
\begin{aligned}
\int_{B}\left(|D x|^{2}+|D y|^{2}\right) d u d v & =\int_{B_{R}\left(x_{0}, y_{0}\right)} \frac{t+A+r+C}{\sqrt{\Delta}} d x d y \\
& \leq C R^{2}+\int_{\partial B_{R}} D z \cdot \nu d \sigma \\
& \leq C\left(R^{2}+R\right),
\end{aligned}
$$

which verifies Assumption (F). Assumption (E) follows from Assumption (C) via

$$
\begin{aligned}
& \omega_{1}(x, y)=\tilde{h}_{1}(x, y)=C_{q}=\phi_{4}, \\
& \omega_{2}(x, y)=h_{1}(x, y)-\tilde{h}_{2}(x, y)=2 B_{q}+C_{p}=\phi_{3}, \\
& \omega_{3}(x, y)=h_{2}(x, y)-\tilde{h}_{3}(x, y)=-A_{q}-2 B_{p}=-\phi_{2}, \\
& \omega_{4}(x, y)=h_{3}(x, y)=A_{p}=\phi_{1} .
\end{aligned}
$$

The estimates $(17,18)$ therefore hold in any disc $B_{\rho}, 0<\rho<1$. In particular, by taking $\rho=1 / 2$,

$$
\begin{gathered}
|D x(0,0)|,|D y(0,0)| \leq C, \\
(D x \wedge D y)(0,0) \geq c .
\end{gathered}
$$

The conformality relations $(5,6,7)$ translate this into the estimate

$$
\left|D^{2} z\left(x_{0}, y_{0}\right)\right| \leq C \text {. }
$$

The Hölder estimate for the second derivatives follows from [7, 9] as required.

\section{ACKNOWLEDGMENT}

It is a pleasure to thank Ronald Gariepy and all participants of the EPSCoR Seminar for their warm hospitality during my stay in Lexington.

\section{REFERENCES}

1. E. Heinz, Über gewisse elliptische Systeme von Differentialgleichungen zweiter Ordnung mit Anwendung auf die Monge-Amperèsche Gleichung, Math Ann. 131 (1956), 411-428.

2. __ Neue a-priori-Abschätzungen für den Ortsvektor einer Fläche positiver Gaußscher Krümmung durch ihr Linienelement, Math. Z. 74 (1960), 129-157.

3. __ Interior estimates for solutions of elliptic Monge-Ampere equations, in Partial Differential Equations, Proceedings of Symposia in Pure Mathematics, vol. IV (Berkeley, 1960), pp. 149-155.

4. __ Über das Nichtverschwinden der Funktionaldeterminante bei einer Klasse eineindeutiger Abbildungen, Math. Z. 105 (1968), 87-89.

5. H. Lewy, On the non-vanishing of the Jacobian in certain one-to-one mappings, Bull. Amer. Math. Soc. 42 (1936), 689-692.

6. __ A priori limitations for solutions of Monge-Ampère equations, Trans. Amer. Math. Soc. 37 (1935), 417-434. 
7. F. Schulz, A priori estimates for solutions of Monge-Ampère equations, Arch. Rational Mech. Anal. 89 (1985), 123-133.

8. __ Univalent solutions of elliptic systems of Heinz-Lewy type, Ann. Inst. Henri Poincaré, Anal. Non Linéaire 6 (1989), 347-361.

9. F. Schulz and L.-Y. Liao, Regularity of solutions of two-dimensional Monge-Ampere equations, Trans. Amer. Math. Soc. 307 (1988), 271-277.

Department of Mathematics, University of Iowa, Iowa City, Iowa 52242 